\title{
Follicular lymphoma grade 3B and diffuse large B-cell lymphoma present a histopathological and molecular continuum lacking features of progression/
} transformation

Karoline Koch, Julia Richter, ${ }^{1}$ Christoph Hänel, ${ }^{1}$ Andreas Hüttmann, ${ }^{2}$ Ulrich Dührsen ${ }^{2}$ and Wolfram Klapper ${ }^{1}$

${ }^{1}$ Department of Pathology, Hematopathology Section, University Hospital SchleswigHolstein, Kiel and ${ }^{2}$ Department of Hematology, University Hospital Essen, University of Duisburg-Essen, Essen, Germany
Correspondence: K. Koch karoline.koch@uksh.de

Received: June 1, 2021. Accepted: $\quad$ November 11, 2021. Prepublished: January 13, 2022.

https://doi.org/10.3324/haematol.2021.279351

(๑2022 Ferrata Storti Foundation Published under a CC BY-NC license @ (ब)

\begin{abstract}
The sole distinguishing feature of follicular lymphoma grade 3B and diffuse large B-cell lymphoma is the growth pattern assessed by histopathology. Diffuse growth defines diffuse large B-cell lymphoma but the clinical relevance of this finding when occurring in follicular lymphoma grade $3 \mathrm{~B}$ is uncertain. To address this issue, individual and coexisting follicular lymphoma grade 3B and diffuse large B-cell lymphoma were separated and analyzed for immunophenotype and molecular genetic features by fluorescence in situ hybridization, targeted sequencing and gene expression profiling. Clinical features of follicular lymphoma grade 3B with and without coexisting diffuse large B-cell lymphoma were studied in homogeneously treated patients from a prospective randomized trial. Follicular lymphoma grade 3B and diffuse large B-cell lymphoma frequently show an intermediate growth pattern and/or occur simultaneously in the same tissue at the time of initial diagnosis. When occurring simultaneously follicular lymphoma grade 3B and diffuse large B-cell lymphoma do not differ significantly for genetic aberrations or phenotype but have distinct gene expression features reflecting a divergent microenvironment. Follicular lymphoma grade 3B with and without coexisting diffuse large B-cell lymphoma do not differ for major clinical parameters such as International Prognostic Index, response to immuno-chemotherapy, progression or overall survival. Follicular lymphoma grade 3B and simultaneous diffuse large B-cell lymphoma are molecularly homogenous. Histological detection of diffuse large B-cell lymphoma is not associated with features of a more aggressive disease and does not reflect transformation or progression of follicular lymphoma grade 3B.
\end{abstract}

\section{Introduction}

Follicular lymphoma (FL) is one of the most frequent lymphoma entities in central Europe and Northern America and is subdivided into "grades" by morphology. Follicular lymphoma grades 1, 2 and 3A are composed of centrocytes and centroblasts and distinguished by centroblast content whereas FL grade 3B (FL3B) consists exclusively of centroblasts. ${ }^{1}$ Since the cytomorphology of FL3B is identical to that of diffuse large B-cell lymphoma (DLBCL), the sole distinguishing feature of FL3B and DLBCL is the growth pattern assessed by histopathology which is follicular in FL3B and diffuse in DLBCL. Once the neoplastic cells in FL3B display areas of diffuse growth the lymphoma fulfills the diagnostic criteria of DLBCL according to the World Health Organization (WHO) classification. Thus, diffuse areas of FL3B are considered "transformation" and this feature has been suggested to be of clinical significance. ${ }^{2,3}$ Unlike FL grades 1, 2 and $3 \mathrm{~A}, \mathrm{FL} 3 \mathrm{~B}$ is considered an aggressive lymphoma by most clinical research groups and treated in the same way as DLBCL ${ }^{4}$ despite the fact that under rituximab-containing poly-chemotherapy (e.g., rituximab, cyclophosphamide, doxorubicin, vincristine, prednisone [R-CHOP]) outcomes might not be very different between FL3A and FL3B. 5,6 Transformation of FL grades 1, 2 and $3 A$ to DLBCL has been shown to be associated with major changes in molecular pathogenic pathways and is well established to reflect a true "transformation" with change in clinical behavior and 
necessity of treatment strategies that may differ from those for $F L 1,2$ and $3 A .^{7}$ In a large retrospective study FL3B seemed to be associated with a favorable outcome. ${ }^{8}$ However, following the current definition of the WHO classification FL3B harboring areas of diffuse growth fulfilling the criteria of DLBCL are rather rare and probably underrepresented ${ }^{5,9}$ or even excluded from analysis in more recent studies on the clinical features of FL3B. ${ }^{6,8}$ It is, therefore, unclear whether histological transition of follicular to diffuse growth reflects a clinically relevant transformation at all. Lack of knowledge regarding the biological and clinical significance of transformation of FL3B may however create a considerable clinical problem since FL3B occurs in patients who are often young - a subgroup of patients who warrant careful consideration of therapy intensity with respect to long-term toxicity. ${ }^{10}$

As FL3B is one of the rarest grades of FL very few molecular studies have been conducted and most of them focused on the distinction of FL3B from other grades and DLBCL. ${ }^{11-14}$ In the current study we aimed to understand the molecular and clinical features of FL3B showing transition into DLBCL in order to provide a definition of "transformation" in FL3B guiding clinical decision-making.

\section{Methods}

\section{Case selection, histological evaluation and immunohistochemistry}

Cases with a diagnosis of FL3B with or without a DLBCL component diagnosed between 2012 and 2016 at the Hematopathology Section and Lymph Node Registry of the University Hospital Schleswig-Holstein, (Kiel, Germany) were identified in the files and re-evaluated with regard to diagnosis and growth patterns as well as suitability for molecular analysis. In total, 51 specimens with a histological picture of FL3B were identified, two of which were excluded later as they turned out to have an IRF4 break and these are considered a separate entity by the WHO classification. ' Staining for CD20 (clone L26, DAKO), CD10 (clone 56C6, Novocastra), Mum1 (clone MUM1P, Dako), Bcl6 (clone BL6.02, DCS) and Bcl2 (clone 100/D5, DBS) was evaluated semiquantitatively (negative, $<25 \%$ of lymphoma cells positive, $<50 \%$ positive, $<75 \%$ positive, $>75 \%$ positive) and proliferative rate was determined according to Ki67 (clone SP6, Neomarkers) in steps of $10 \%$ by visual inspection. Immunohistochemical classification of the cell of origin of DLBCL components was performed applying the Hans classifier. ${ }^{15}$ Areas of defined growth patterns were selected for tissue microarray construction in 47 cases with sufficient material. Meshworks of follicular dendritic cells were evaluated in tissue microarrays for each growth pattern semiquantitatively by CD21 staining ( $0=$ absence of meshworks, 1 = markedly reduced covering $<50 \%$ of fol- licles, 2 = slightly reduced covering $>50 \%$ of follicles and 3 = intact covering whole area of follicle; clone 2G9, Novocastra). Punches with a tissue microarray needle $(1 \mathrm{~mm}$ diameter) from each area were also used for nucleic acid extraction. To prevent cross-contamination of DNA/RNA analytes the tissue microarray needle was punched three to five times in an empty paraffin block between each punching of a patient's specimen.

The study was conducted in accordance with the recommendations of the ethics board of the Medical Faculty, University of Kiel (D447/10) for the use of archival tissue specimens. Informed consent was obtained from patients treated in the PETAL trial (see below).

\section{Fluorescence in situ hybridization}

Fluorescence in situ hybridization (FISH) was performed on $5 \mu \mathrm{m}$ slides from the tissue microarrays, allowing separate analysis of different growth patterns. Probes for IRF4, $B C L 2, B C L 6$ and MYC were obtained from ZytoVision (Bremerhaven, Germany) and applied as previously described. ${ }^{16}$

\section{DNA and RNA extraction}

Cases for molecular genetic analyses were chosen when the tissue quality was assumed to be sufficient for nucleic acid extraction. Simultaneous DNA and RNA extraction was done using the AllPrep DNA/RNA FFPE Kit (Qiagen) according to the manufacturer's instructions. For each patient different growth patterns were extracted separately from tissue microarray needle biopsies taken from the respective areas (2-3 biopsies per specimen).

\section{Mutation analyses}

Mutation analyses were done on DNA isolated from the formalin-fixed paraffin-embedded material of the diagnostic biopsy using an Ampliseq Custom DNA Panel covering genes or mutational hotspots known to be recurrently mutated in aggressive B-cell lymphomas (Online Supplementary Table S1). Libraries were prepared according to the manufacturer's instructions and sequencing was performed on a MiSeq instrument using V3 sequencing chemistry. FASTQ data were analyzed using JSI SeqNext software (JSI Medical Systems GmbH, Ettenheim, Germany). Formalinfixed paraffin-embedded tissue from reactive lymph nodes from eight healthy individuals (a cancer infiltration was excluded by expert hematopathologists) was used as control tissue, and sequence alterations identified in those samples were subtracted from those of the lymphoma patients. Furthermore, common single nucleotide polymorphisms with $\geq 1 \%$ variant allele frequency were excluded. Potential protein changing alterations with at least $15 \%$ variant allele fraction were analyzed in more detail using the ENSEMBL variant effect predictor (http://www.ensembl.org/Homo_sapiens/Tools/VEP). However, as the library preparation process contains polymerase chain reaction amplification 
steps to enrich the target regions no interpretation of the variant allele fractions was possible. Furthermore, no germline material was available from those samples in order to be able to differentiate between somatic and germline variants. To overcome this limitation, variants were categorized based on the variant effect prediction by SIFT, PolyPhen, FATHMM and Condel into six different groups: (i) high impact; (ii) moderate impact and deleterious or damaging effect; (iii) moderate impact but mixed effect prediction (deleterious/damaging or tolerated based on the different tools used); (iv) moderate impact and tolerated or benign; ( $v$ ) moderate impact but no additional information; and (vi) low impact. Variants with high impact, moderate/deleterious effect prediction and moderate/mixed effect prediction were considered to affect protein function negatively.

\section{Gene expression analysis}

Gene expression was analyzed as previously described applying NanoString technology and the PanCancer Immune Profiling Panel. ${ }^{17}$ Background thresholding and normalization were performed by the NSolver software (version 4.0; NanoString Technologies). Twenty housekeeping genes were chosen for normalization in a two-step process: (i) housekeeping genes with average counts $<100$ were excluded; and (ii) 20 housekeeping genes with the lowest count variability were chosen for normalization. Endogenous genes with normalized expression levels below the calculated background threshold in $>20 \%$ of the analyzed specimens were excluded from further analysis. Fold changes between follicular and diffuse growth patterns were calculated for each individual case. Genes with a fold change of $>1.2$ or $<-1.2$ in at least $50 \%$ of cases were chosen and mean fold changes of these genes were calculated as fold change of the geometric means of the respective single case gene expression levels. Genes with a mean fold change of $>1.5$ or $<-1.5$ were considered to be differentially expressed.

\section{Clinical analysis}

The features of patients with FL3B $(n=17)$ and FL3B+DLBCL $(n=16)$ were analyzed in patients treated in the prospective randomized 'Positron Emission Tomography-Guided Therapy of Aggressive Non-Hodgkin Lymphomas' trial (PETAL: clinicaltrials.gov NCT00554164 and EudraCT 2006-00164133). ${ }^{18}$ Patients received two cycles of standard R-CHOP followed by interim $\left[{ }^{18} \mathrm{~F}\right]$ fluorodeoxy-glucose-positron emission tomography (PET). Interim PET-negative patients continued R-CHOP whereas interim PET-positive patients were randomized between continued R-CHOP and an intensive methotrexate- and cytarabine-based Burkitt lymphoma protocol. According to current treatment guidelines ${ }^{19}$ all patients were treated at the timepoint of first diagnosis.

\section{Statistical analysis}

Statistical analysis was performed using GraphPadPrism (version 7.00 for Windows, GraphPad software, La Jolla, CA, USA) applying the $\chi^{2}$ test, Fisher exact test and unpaired $t$ test as indicated in the Results section. Kaplan-Meier curves were generated using SPSS statistics (version 26.0, IBM, Armonk, NY, USA).

\section{Results}

\section{Growth pattern of follicular lymphoma grade 3B with a continuum to diffuse large B-cell lymphoma}

Forty-nine lymphomas with a diagnosis FL3B were reanalyzed for growth pattern. All cases fulfilled the criteria of FL3B and 27/49 (55\%) additionally harbored areas with diffuse growth representing $D L B C L$ by definition (FL3B+DLBCL). None of the cases included any low-grade FL component. None of the cases showed the typical constellation of pediatric type FL according to the current WHO classification. ${ }^{1}$ Relapse biopsies were available for two patients both of whom had FL3B+DLBCL at primary diagnosis and DLBCL at relapse. The proportion of DLBCL ranged between $10 \%$ and $95 \%$ in individual cases (mean: $49 \%$ ). The cytomorphology of the DLBCL component was centroblastic in $25 / 27$ cases and immunoblastic in $2 / 27$ cases. In all cases, the morphology of the DLBCL component matched the morphology of the FL3B component. Within the areas of follicular growth two different growth patterns were noticed: (i) clearly demarcated, roundish follicles that were separated from each other (follicular pattern) (Figure 1) and (ii) follicles localized close to each other appearing to merge (confluent pattern) (Figure 1). The confluent pattern was clearly distinguishable from a diffuse pattern/DLBCL in hematoxylin and eosin staining and could be further highlighted by staining for $T$ cells and follicular dendritic cells (Figure 1). Of the 22 pure FL3B lacking an additional DLBCL component, only four cases showed a pure follicular pattern (4/22, 18\%), whereas $11 / 22$ (50\%) cases solely displayed the confluent pattern. A combination of both patterns was detectable in $7 / 22(32 \%)$ of pure FL3B. The distribution of these growth patterns within the FL3B component was different in FL3B+DLBCL cases (confluent pattern 14/27 [52\%], follicular pattern $7 / 27$ [26\%] and a combination of both patterns 6/27 [22\%], $P=0.0421, \chi^{2}$ test) although the differences must be interpreted with caution because of the small number of cases. All growth patterns and their combinations are indicated in Table 1.

\section{Homogeneous immunophenotype in follicular lymphoma grade 3B and diffuse large B-cell lymphoma}

CD20 was homogeneously expressed in all 49 cases. CD10 expression was more variable with $22 / 49$ cases (45\%) being completely negative, $4 / 49$ cases ( $8 \%$ ) with ex- 
pression in $<50 \%$ of cells and $23 / 49$ cases (47\%) with expression in $>75 \%$ of cells. Bcl 2 staining was available for 48 cases. Bcl 2 was completely negative in $5 / 48$ cases (10\%), positive in $<50 \%$ of cells in $7 / 48$ cases $(15 \%)$ and positive in $>50 \%$ of cells in $36 / 48$ cases $(75 \%)$. Staining for Mum1 was done in 36 cases. Mum1 was completely negative in $3 / 36$ cases ( $8 \%$ ), positive in $<25 \%$ of cells in $9 / 36$ cases (25\%), positive in $25 \%$ to $<50 \%$ of cells in $4 / 36$ cases $(11 \%)$, positive in $50 \%$ to $<75 \%$ of cells in $11 / 36$ cases (31\%) and positive in $\geq 75 \%$ of cells in $9 / 36$ cases (25\%). Bcl6 staining was available for 29 cases. Of these, seven cases $(24 \%)$ were positive in $50 \%$ to $<75 \%$ of cells and 22 cases $(76 \%)$ were positive in $\geq 75 \%$ of cells. The proliferative rate by Ki67 ranged between $30 \%$ and $90 \%$ with a mean proliferative rate of $63 \%$ in the whole cohort. Classification of the cell of origin was available for 26 cases with a DLBCL component. Of these, 19 cases (73\%) displayed a germinal center B-cell-phenotype and seven cases (27\%) displayed a non-germinal center B-cell-phenotype according to the Hans classifier..$^{15}$ Despite intertumoral variability, expression of the above-mentioned antigens did not differ between different growth patterns within an individual patient/lymphoma. Of note, FL3B and

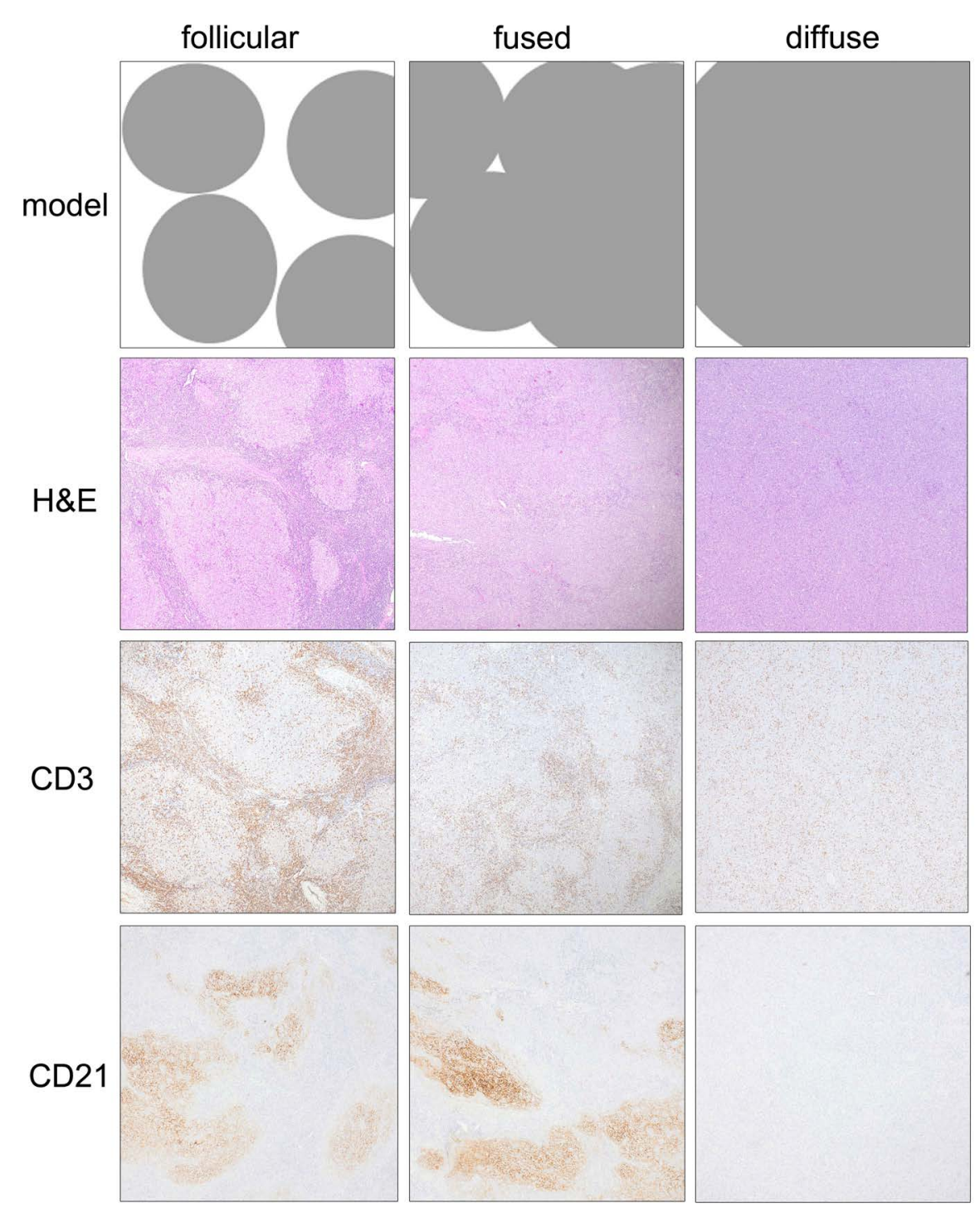

Figure 1. Growth pattern of follicular lymphoma grade 3B and diffuse large B-cell lymphoma. The three patterns observed are shown for exemplary cases in the three columns as a scheme/model, hematoxylin and eosin staining (H\&E) and staining for CD3 and CD21. Original magnification 40x.
DLBCL areas in the same specimen showed the same proliferative rate according to Ki67 (Figure 2). Comparing FL3B and FL3B+DLBCL, no significant differences between mean proliferative rates were detectable $(59 \%$ and $65 \%$, respectively, $P=0.1349$, unpaired $t$-test). $C D 21$ staining was evaluable in 12 follicular FL3B components, 26 confluent FL3B components and 16 DLBCL components of 34 cases. Meshworks of follicular dendritic cells showed considerable intercase variability. The highest scores were reached in follicular FL3B (8/12, 67\% slightly reduced/intact and 4/12, 33\% markedly reduced); DLBCL components mostly showed markedly reduced or absent meshworks (1/16, 6\% slightly reduced and 15/16, 94\% markedly reduced/absent). Confluent FL3B presented intermediate stages of follicular dendritic cell preservation (14/26, 54\% slightly reduced/intact and 12/26, 46\% markedly reduced/absent meshworks), supporting the idea that confluent FL3B represents an intermediate stage between follicular FL3B and DLBCL. Within individual cases with evaluable FL3B and DLBCL components $(n=14)$, a reduction of meshworks could be observed between the FL3B and DLBCL components in $9 / 14$ cases (64\%) and the same stage of preservation in $5 / 14$ cases (36\%).

\section{Chromosomal aberrations are mostly stable between follicular lymphoma grade 3B and diffuse large B-cell lymphoma}

Lymphomas with breaks in IRF4 were excluded since these are considered a distinct entity in the WHO classification. FISH to detect breaks in BCL2, BCL6 and MYC was performed for 40 cases, 21 of which contained a DLBCL component. In any lymphoma containing multiple growth patterns these were analyzed separately. Overall, 27 chromosomal breaks were detectable in 25 cases and 15 cases did not show breaks in the above-mentioned genes. One case presented a double-hit constellation with $M Y C$ and $B C L 6$ rearrangements (FL3B with confluent pattern). Chromosomal breaks occurred at a similar frequency in FL3B and

Table 1. Coexistence of growth patterns in follicular lymphoma grade $3 B$.

\begin{tabular}{|l|c|c|c|c|}
\hline & Follicular & Confluent & Diffuse & Total \\
\hline FL3B (N=22) & + & - & - & $4 / 22(18 \%)$ \\
& - & + & - & $11 / 22(50 \%)$ \\
\hline $\begin{array}{l}\text { FL3B/DLBCL } \\
\text { (N=27) }\end{array}$ & + & + & - & $7 / 22(32 \%)$ \\
& - & - & + & $7 / 27(26 \%)$ \\
& + & + & + & $14 / 27(52 \%)$ \\
& + & + & + & $6 / 27(22 \%)$ \\
\hline
\end{tabular}

Numbers and percentages correspond to the respective subgroup. FL3B: follicular lymphoma grade 3B; DLBCL: diffuse large B-cell lymphoma. 

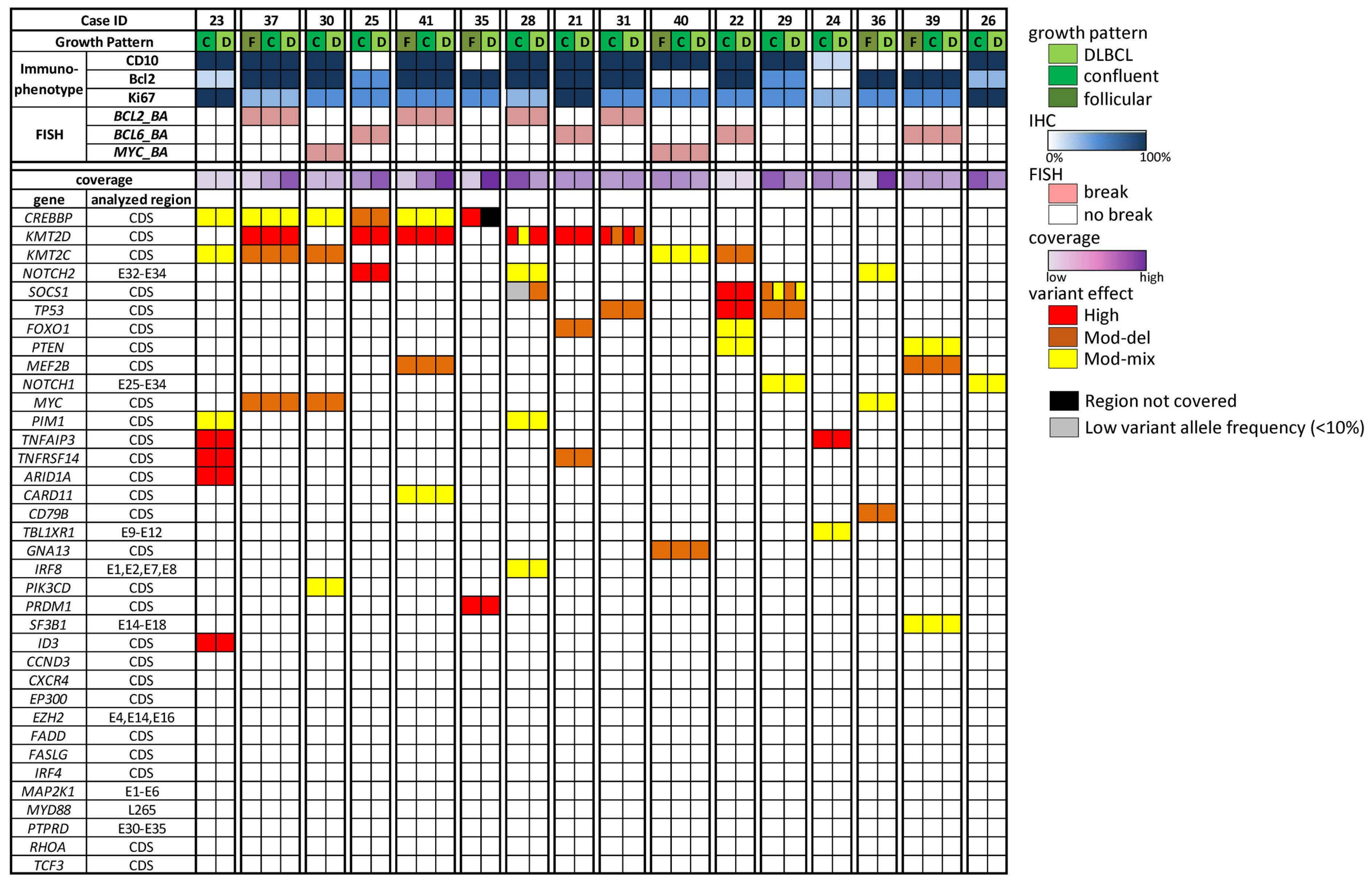

Figure 2. Mutational pattern of the different growth patterns of follicular lymphoma grade 3B + diffuse large B-cell lymphoma. Within each lymphoma the patterns were analyzed separately. Individual cases are indicated by the case identifie (ID) and growth pattern as D: diffuse large B-cell lymphoma (DLBCL) component; F: follicular component; C: confluent component. The immunophenotype was scored in a five-tiered way 0\%; 1-25\%; $26-50 \% ; 51-75 \% ;>75 \%$ positive tumor cells and is displayed as a color code as shown in the scale bar. Fluorescence in situ hybridization (FISH) was done using break apart probes (BA). Potential protein-changing variants identified in the different components were colored according to the variant effect prediction (red: high; dark orange: moderate, deleterious; yellow: moderate, deleterious and tolerated effect based on different algorithms used, black: region was not covered in the DLBCL sample, gray: variant was identified with low variant allele frequency (<10\%) in the confluent component. If more than one variant with different effects targeting one gene was identified in one component both variant effects are shown separately. E: exon; CDS: coding region; \#: region was not covered in the DLBCL component of that sample. \$: variant was identified with low $(<10 \%)$ variant allele frequency in the confluent component. IHC: immunohistochemistry.

FL3B+DLBCL (FL3B: BCL2 in 2/19 [11\%], BCL6 in 8/19 [42\%], MYC in 1/19 [5\%]; FL3B+DLBCL: BCL2 in 6/21 [29\%], BCL6 in $7 / 21$ [33\%], MYC in 3/21 [14\%], $P=0.2409$ for $B C L 2, P>0.9999$ for $B C L 6, P=0.6094$ for $M Y C$, Fisher exact test). FISH results for at least two growth patterns of the same lymphoma were available for 21 cases. In most cases the chromosomal aberrations were shared between the different growth patterns (20/21 cases, 95\%). However, in $1 / 21(5 \%)$ cases a divergent result was observed concerning $B C L 6$. This case harbored a BCL6 break in the confluent area but not in the follicular areas of a FL3B (data not shown).

\section{The microenvironmental composition of follicular lymphoma grade 3B and diffuse large B-cell lymphoma differs}

In order to understand whether the growth patterns rep- resent molecular progression/evolution of the disease, gene expression was analyzed in FL3B+DLBCL cases. Assuming that the follicular pattern and DLBCL represent the two ends of a spectrum of growth patterns in each individual lymphoma, these areas were analyzed separately in cases with both components available $(n=6)$. Since DLBCL are known to be a heterogeneous disease with their molecular features differing from patient to patient, we analyzed differential expression within each patient comparing the follicular FL3B with the DLBCL component in cases with both components available. Using the criteria described in the Methods section we identified a low number of genes $(n=45)$ differentially expressed in multiple patients (Online Supplementary Figure S1). Of these, $33 / 45$ (73\%) genes were more highly expressed in the follicular FL3B area and reflected the expression pat- 
tern of the germinal center microenvironment, such as follicular dendritic cells (CD21), follicular T helper cells (ICOS, STAT4), T cells (CD3, TCF7, TXK) and CXCL12, which has previously been described to be upregulated in FL stromal cells. ${ }^{20}$ Furthermore, genes more highly expressed in follicular areas reflected a greater abundance of cells of innate immunity (C1QB, SIGLEC1, TPSAB1) including natural killer cells (KLRB1, KLRG1) compared to the DLBCL component. In line with a higher content of immune cells, genes involved in trafficking e.g. by adhesion and motility, were more highly expressed in follicular areas (CCR7 and its ligand CCL21, CDH1, ITGA6, ITGB6). Alterations of the extracellular matrix between follicular and diffuse areas are reflected by differential expression of collagen III $\alpha$ and fibronectin which were more highly expressed in the DLBCL component. Of note, two of 12 genes more highly expressed in the diffuse part are known to be involved in T-cell activation (CD7O and TNFSF4/OXL4O). Only a few of the differentially expressed genes could be assigned to Bcell differentiation (e.g. CD27) suggesting that the gene expression pattern of the neoplastic $B$ cells does not differ substantially between follicular (FL3B) and diffuse (DLBCL) areas. No differences were observed in genes described to be associated with transformation or an aggressive phenotype of FL (MYC, NOTCH, NFkB signaling pathway genes, data not shown).

\section{Molecular genetic features are stable between follicular lymphoma grade 3B and diffuse large B-cell lymphoma}

Genes recurrently mutated in mature B-cell lymphoma were analyzed by targeted sequencing in pure FL3B $(n=11)$ and FL3B+DLBCL $(n=16)$. The FL3B+DLBCL group consisted of two cases containing follicular and diffuse components, ten cases with a confluent and a diffuse component and four cases with all growth patterns in the same specimen. Areas representing growth patterns were separated by macrodissection and analyzed individually (yielding 36 sequencing results). Sequencing retrieved a mean read depth of 4,258 ( $\min 276, \max 10,131$ ). Overall, 81 genetic variants were detected (Online Supplementary Table S2). The genetic variants were mostly missense variants $(n=56)$, followed by nonsense $(n=9)$, and splice site or splice region variants $(n=8)$, frameshift $(n=4)$ and inframe variants $(n=4)$. The pure FL3B group consisted of nine cases with a confluent growth pattern, one case with a follicular growth pattern and one case with a follicular and confluent growth pattern of which only the confluent component was analyzed. Sequencing retrieved a mean read depth of 5,146 ( $\mathrm{min} 967$, max 9,769). Overall, 22 genetic variants were detected (Online Supplementary Table S2). The genetic variants were missense in most cases $(n=14)$, followed by nonsense $(n=7)$ and splice site or splice region variants $(n=1)$. Since germline DNA was not available, variant effect prediction using ENSEMBLE was performed to estimate the functional rel- evance of the genetic alterations. In the FL3B+DLBCL group, variant effect prediction identified 16/81 (20\%) variants with high impact, $17 / 81$ (21\%) with moderate/deleterious effects, $29 / 81$ (36\%) with moderate/mixed effects, $13 / 81$ (16\%) with moderate/tolerated or unclear effects and 6/81 (7\%) with low impact. In the pure FL3B group, 9/22 (41\%) variants were predicted to have high impact, $6 / 22$ (27\%) with moderate/deleterious effects, $4 / 22$ (18\%) with moderate/mixed effects and $3 / 22$ (14\%) with moderate/tolerated effects. The number of variants detected per case was significantly different between pure FL3B ( 0 to 6 ; mean 2) and FL3B+DLBCL (1 to 11; mean 5.1; $P=0.0037$, unpaired $t$-test). Overall, 62/81 (77\%) variants in FL3B+DLBCL and 19/22 (86\%) variants in pure FL3B were predicted to affect protein function negatively. Considering only variants with potentially negative effects on protein function, significant differences between FL3B and FL3B+DLBCL could still be observed (FL3B: 0 to 6 variants per case, mean 1.7 and FL3B+DLBCL: 1 to 8 variants per case, mean 3.9; $P=0.0091$, unpaired $t$-test). However, as a targeted sequencing approach was applied, this does not allow conclusions on the whole load of genetic variants in individual tumors. The most frequently altered genes with potentially negative effects on protein function were KMT2D (6/16 cases, 38\%), CREBBP (6/16 cases, 38\%) and KMT2C (5/16 cases, $31 \%)$ in FL3B+DLBCL and TP53 (3/11 cases, 27\%), MYD88 (2/11 cases, $18 \%)$ and $K M T 2 D(2 / 11$ cases, $18 \%)$ in pure FL3B. No significant differences were found between FL3B and $\mathrm{F} L 3 B+D L B C L$ considering variants in specific genes (Online Supplementary Table S4).

In 14/16 (88\%) cases of FL3B+DLBCL all variants were shared between the different specimens/areas of the corresponding sample. Thus, overall $77 / 81$ (95\%) variants did not differ between FL3B and DLBCL occurring in the same patient (Figure 2). In only two cases, four variants were detected that differed between FL and DLBCL within the same patient. In one sample (case 35 ) a variant in the CREBBP gene was detected in the follicular component but no sequencing result was obtained for the DLBCL component due to low coverage. In the other sample (case 28), three variants were discrepant between the FL3B (confluent pattern) and the DLBCL component. Two variants affecting NOTCH1 and SOCS1 were identified in the confluent and the DLBCL component, respectively; both had a very low frequency ( $<10 \%$ of reads) in the other component and were therefore initially not considered. In contrast, a KMT2D mutation was present in the FL3B and completely absent in the DLBCL component (Figure 2). This variant was predicted to have a moderate/mixed effect.

\section{The clinical features of follicular lymphoma grade 3B alone and with diffuse large B-cell lymphoma do not differ}

To understand whether the co-occurrence of DLBCL with 
FL3B is associated with clinical progression, we analyzed the subgroup of $F L 3 B$ in the prospective randomized PETAL trial. ${ }^{18}$ In this clinical cohort 17 patients were classified as having FL3B and 16 as having FL3B+DLBCL. All the patients with $F L 3 B$ and $15 / 16$ of those with FL3B+DLBCL had a favorable interim PET scan and were treated with six cycles of R-CHOP, while one FL3B+DLBCL patient had an unfavorable interim $\mathrm{PET}$ scan and received two cycles of R-CHOP followed by the Burkitt lymphoma protocol. The presence of the confluent pattern described in the current manuscript was not assessed by central pathology review in PETAL and is thus not available for further analysis. The baseline characteristics of the FL3B and $F L 3 B+D L B C L$ cohorts did not differ for parameters known to be associated with aggressive disease such as advanced stage, increased lactate dehydrogenase level or International Prognostic Index (Table 2, comparison with DLBCL treated in PETAL in Online Supplementary Table S3). Moreover, we did not find any significant difference in overall treatment response and overall survival (Table 2).
As a consequence, progression-free survival and overall survival did not differ between FL3B and FL3B+DLBCL (Figure 3).

\section{Discussion}

FL3B is a rare subtype of FL, underrepresented in clinical and translational research studies. ${ }^{21}$ The definition of FL3B is exclusively based on histological features: (i) differentiation arrest of neoplastic germinal center cells as centroblasts leading to absence of centrocytes and (ii) follicular growth. The latter is the sole feature distinguishing FL3B from DLBCL. Follicular growth describes an arrangement of lymphoma cells in roundish accumulations of cells which contain microenvironmental structures of physiological germinal centers such as follicular dendritic cells, follicular T-helper cells and occasionally follicle mantle cells. ${ }^{22}$ These organoid arrangements resemble physiological germinal centers more closely in low-grade FL such as

Table 2. Clinical characteristics and treatment response of follicular lymphoma grade 3B with or without diffuse large B-cell lymphoma in patients treated in the prospective randomized 'Positron Emission Tomography-Guided Therapy of Aggressive NonHodgkin Lymphomas' (PETAL) trial. ${ }^{18}$

\section{FL3B alone}

Number of patients

Median age (range)

Baseline characteristics

Male sex

Age $\geq 60$ years

ECOG performance status $>1$

Lactate dehydrogenase $>$ ULN

Ann Arbor stage III or IV

Extranodal manifestations $>1$

Bone marrow infiltration

B symptoms

International Prognostic Index

$$
\text { Low }
$$

Low-intermediate

High-intermediate

High

Treatment response
17

51 years $(29-72)$

\section{FL3B with DLBCL}

16

57 years (29-76)

\begin{tabular}{|l|l|l|l|l|l|}
\hline Overall response & 16 & 94.1 & 15 & 93.8 & $>0.9999$ \\
\hline Complete remission & 12 & 70.6 & 10 & 62.5 & $>0.9999$ \\
\hline
\end{tabular}

$P$-values according to the Fisher exact test and for the International Prognostic Index by the $\chi^{2}$ test. FL3B: follicular lymphoma grade 3B; DLBCL: diffuse large B-cell lymphoma; ECOG:, Eastern Cooperative Oncology Group; ULN, upper limit of normal. 
FL grades 1 and 2 compared to $F L$ grade $3 A$ and FL3B. Initially, the grades of FL were thought to reflect a multistep process of tumor progression in analogy to grades of differentiation in solid tumors. ${ }^{23}$ However, there are several findings suggesting that at least a large fraction of FL3B cases are not a progressive form of $F L$ grades 1, 2 and $3 A$. First, unlike FL grades 1, 2 and $3 A, F L 3 B$ is diagnosed more frequently in younger patients, suggesting a unique pathogenesis. ${ }^{10,21}$ Second, molecular studies, e.g. on chromosomal translocations show, a much lower frequency of aberrations involving $B C L 2$ and a higher frequency of $B C L 6$ translocations in FL3B than in FL grades 1, 2, and 3A.9,11,13,24
Third, FL3B often co-exists with a DLBCL at initial diagnosis whereas transformation of indolent $F L$ grades 1, 2 and $3 \mathrm{~A}$ mostly occurs at a later stage of the disease., Fourth, FL3B rarely follows or co-exists with FL grades 1, 2 and $3 A$, again suggesting differences in pathogenic pathways. $^{9}$

In the current study we analyzed an aspect of FL3B biology not addressed by previous studies. We aimed to understand whether the loss of follicular growth indicates molecular and clinical progression/transformation. To the best of our knowledge, this is the first study specifically analyzing the intratumoral heterogeneity of $F L 3 B$ and
A

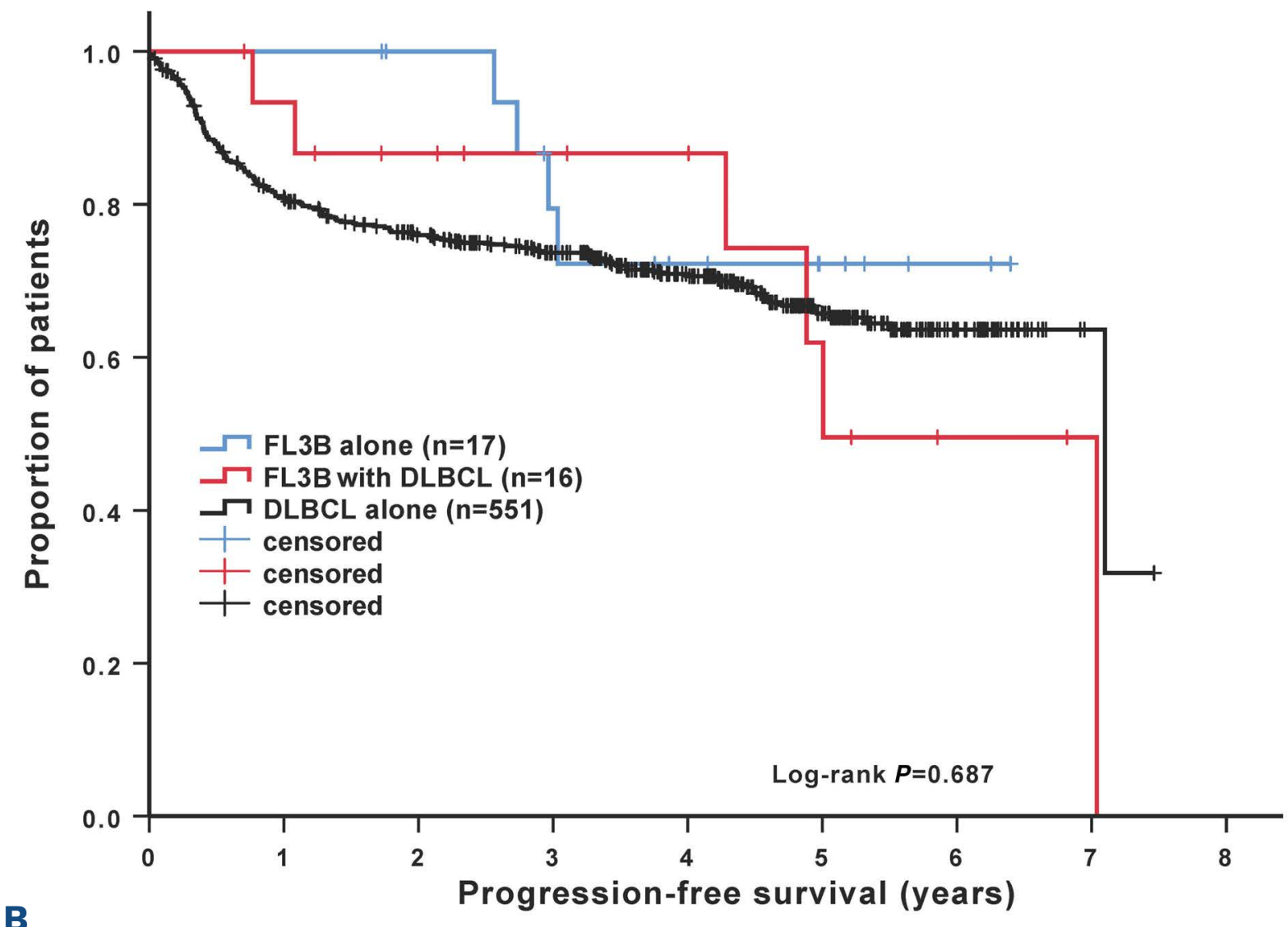

B

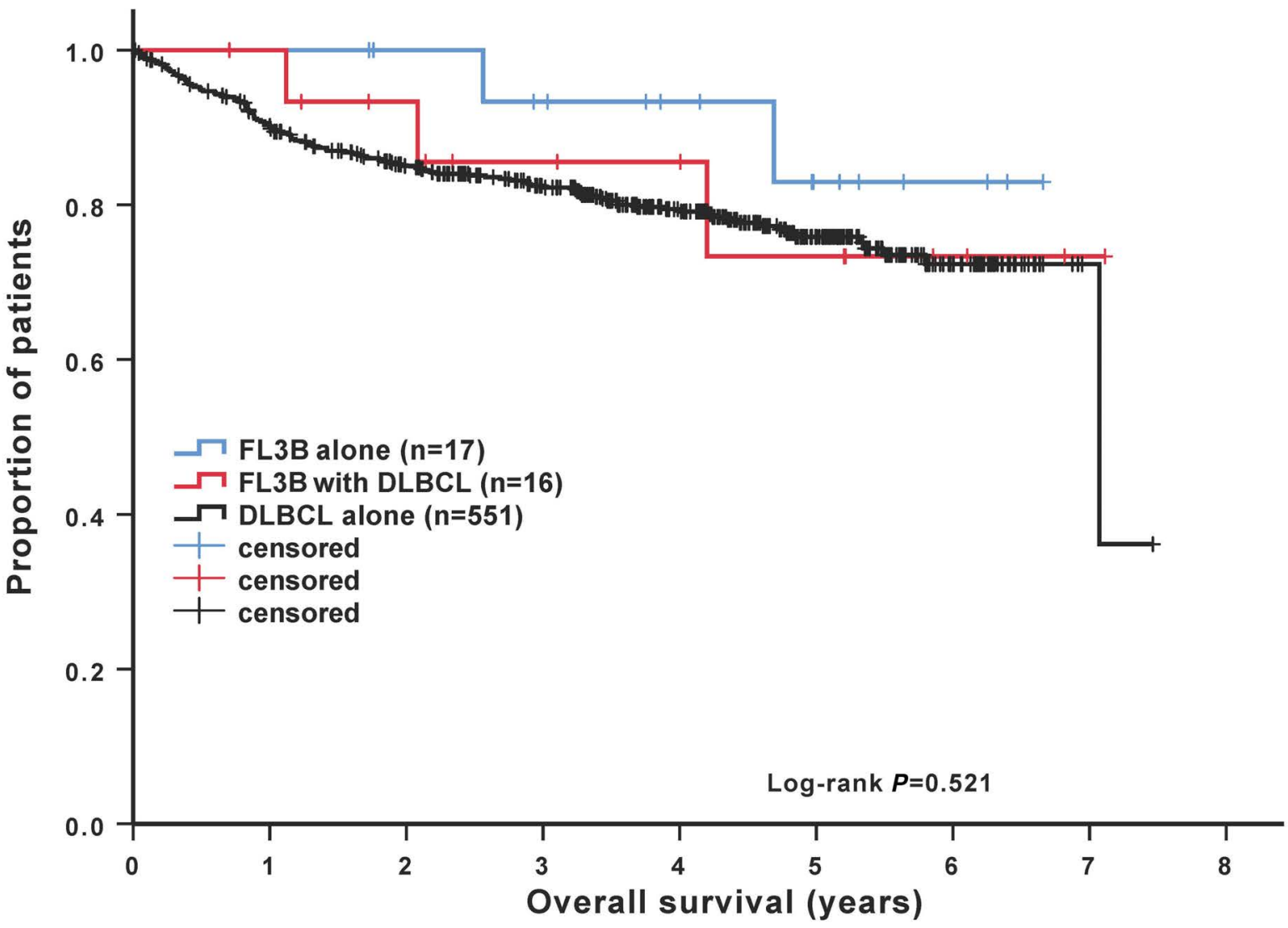

Figure 3. Kaplan-Meier survival estimates for progression free (A) and overall survival $(B)$ of patients with follicular lymphoma grade 3B with or without diffuse large $B$ cell lymphoma. Patients with diffuse large B-cell lymphoma (DLBCL) treated in the same trial are shown as a control (see Online supplementary Table S3). As patients with activated B-cell or germinal center B-cell DLBCL did not differ significantly regarding progression-free survival and overall survival these subgroups were not differentiated. 
DLBCL within individual patients/lymphomas. Similarly to previous studies, ${ }^{5,9}$ we found that a large number of cases of FL3B were associated with DLBCL at first diagnosis. Moreover, we found a pattern intermediate between follicular and diffuse growth endorsing the impression of a pathological continuum between FL3B and DLBCL. Furthermore, we showed that the molecular features of coexisting FL3B and DLBCL were homogeneous and that there was no molecular evidence of progression/transformation. In fact, the differences between follicular and diffuse growth - representing FL3B and DLBCL, respectively - were subtle and restricted to the features of the non-neoplastic microenvironment. Considering these findings it was not surprising to see that clinical features did not differ between patients with FL3B and those with FL3B+DLBCL. It seems reasonable to assume that FL3B represents a molecular and clinical continuum with DLBCL. Thus, FL3B+DLBCL does not reflect progression or transformation of the disease, suggesting that treatment should follow the same guidelines for FL3B and DLBCL. Of note, the subtype of IRF4 translocation-positive lymphomas now recognized by the WHO classification was excluded from our analysis. However, large B-cell lymphoma with IRF4 translocations shares features with FL3B such as histology (mostly presenting as FL3B and/or DLBCL), ${ }^{25}$ patients' age (younger adults) ${ }^{10,26}$ and outcome (favorable). ${ }^{27}$ To what extent FL3B lacking IRF4 aberrations and large B-cell lymphoma with IRF4 translocations may in fact share pathogenic features needs to be addressed in future studies that might help to specify diagnostic criteria. Another interesting group of patients to analyze in the future might be those with FL3B at primary diagnosis and DLBCL at relapse. However, this is hindered by the fact that only a small proportion of patients with FL3B relapse and of those who do only few undergo a second biopsy. This also holds true for our cohort, in which a second biopsy was only available for two patients. Moreover, a more detailed molecular analysis of FL3B and
FL3B+DLBCL, such as that applied in our current study, may provide a molecular genetic definition of FL3B independent of the histological detection of follicular growth. One could speculate that a molecular definition of FL3B might identify a molecular counterpart among DLBCL lacking a histopathologically detectable FL3B component but sharing clinical features.

\section{Disclosures}

$A H$ has received travel expenses from Celgene and Roche. UD has received research funding from Amgen; has received honoraria and research funding from Celgen and Roche; has acted as a consultant for and received honoraria from AbbVie and Gilead; and has received honoria from Janssen. WK has received honoraria and research funding from Amgen, Regeneron, Hoffman-La Roche and Takeda.

\section{Contributions}

$K K$ and $W K$ designed the research project, $K K, C H$ and $J R$ generated and analyzed pathological and molecular data, $A H$ and UD analyzed clinical data. KK, JR and WK wrote the manuscript. All authors read and agreed on the final version of the manuscript.

\section{Acknowledgments}

The authors would like to thank Dana Germer, Charlotte Botz von Drathen, Reina Zühlke-Jenisch and Lorena Vallés Uriarte for excellent technical support.

\section{Funding}

This work was supported by an intramural grant of the Medical Faculty of the University of Kiel to KK (F359991). The PETAL trial is funded by Deutsche Krebshilfe (grants $n$. 107592 and 110515), Amgen Germany, and Roche Pharma.

\section{Data-sharing statement}

Data will be shared according to ethical and administrative guidelines upon request for collaboration.

\section{References}

1. WHO Classification of Tumors of Haematopoietic and Lymphoid Tissues. Revised 4th ed: International Agency for Reaserch on Cancer, 2018.

2. Hans CP, Weisenburger DD, Vose JM, et al. A significant diffuse component predicts for inferior survival in grade 3 follicular lymphoma, but cytologic subtypes do not predict survival. Blood. 2003;101(6):2363-2367.

3. Ganti AK, Weisenburger DD, Smith LM, et al. Patients with grade 3 follicular lymphoma have prolonged relapse-free survival following anthracycline-based chemotherapy: the Nebraska Lymphoma Study Group experience. Ann Oncol. 2006;17(6):920-927.

4. Dreyling M, Ghielmini M, Rule S, et al. Newly diagnosed and relapsed follicular lymphoma: ESMO clinical practice guidelines for diagnosis, treatment and follow-up. Ann Oncol. 2021;32(3):298-308.

5. Mustafa Ali M, Rybicki L, Nomani L, et al. Grade 3 follicular lymphoma: outcomes in the rituximab era. Clin Lymphoma Myeloma Leuk. 2017;17(12):797-803.

6. Shustik J, Quinn M, Connors JM, Gascoyne RD, Skinnider B, Sehn LH. Follicular non-Hodgkin lymphoma grades $3 \mathrm{~A}$ and 3B have a similar outcome and appear incurable with anthracycline-based therapy. Ann Oncol. 2010;22(5):1164-1169.

7. Kumar E, Pickard L, Okosun J. Pathogenesis of follicular lymphoma: genetics to the microenvironment to clinical translation. Br J Haematol. 2021;194(5):810-821.

8. Wahlin BE, Yri OE, Kimby E, et al. Clinical significance of the WHO grades of follicular lymphoma in a population-based 
cohort of 505 patients with long follow-up times. $\mathrm{Br} \mathrm{J}$ Haematol. 2012;156(2):225-233.

9. Koch K, Hoster E, Ziepert M, et al. Clinical, pathological and genetic features of follicular lymphoma grade 3A: a joint analysis of the German Low-Grade and High-Grade Lymphoma Study Groups GLSG and DSHNHL. Ann Oncol. 2016;27(7):1323-1329.

10. Paul U, Richter J, Stuhlmann-Laiesz C, et al. Advanced patient age at diagnosis of diffuse large B-cell lymphoma is associated with molecular characteristics including $A B C$-subtype and high expression of MYC. Leuk Lymphoma. 2018;59(5):1213-1221.

11. Horn $\mathrm{H}$, Schmelter $\mathrm{C}$, Leich $\mathrm{E}$, et al. Follicular lymphoma grade $3 \mathrm{~B}$ is a distinct neoplasm according to cytogenetic and immunohistochemical profiles. Haematologica. 2011;96(9):1327-1334.

12. Piccaluga PP, Califano A, Klein U, et al. Gene expression analysis provides a potential rationale for revising the histological grading of follicular lymphomas. Haematologica. 2008;93(7):1033-1038.

13. Bosga-Bouwer AG, van Imhoff GW, Boonstra R, et al. Follicular lymphoma grade $3 B$ includes 3 cytogenetically defined subgroups with primary $\mathrm{t}(14 ; 18), 3 \mathrm{q} 27$, or other translocations: $t(14 ; 18)$ and $3 q 27$ are mutually exclusive. Blood. 2003;101(3):1149-1154.

14. Horn H, Kohler C, Witzig R, et al. Gene expression profiling reveals a close relationship between follicular lymphoma grade $3 \mathrm{~A}$ and $3 \mathrm{~B}$, but distinct profiles of follicular lymphoma grade 1 and 2. Haematologica. 2018;103(7):1182-1190.

15. Hans CP, Weisenburger DD, Greiner TC, et al. Confirmation of the molecular classification of diffuse large B-cell lymphoma by immunohistochemistry using a tissue microarray. Blood. 2004;103(1):275-282.

16. Au-Yeung KHR, Padilla LA, Burkhardt B, Woessmann W, Klapper W. Features of pediatric B-lymphoblastic lymphoma with KMT2A rearrangements in the German NHL-BFM group. The 108th Annual Meeting of the Japanese Society of Pathology. Tokyo: Proceedings of the Japanese Society of Pathology, 2019:420. 17. Reinke S, Bröckelmann PJ, laccarino I, et al. Tumor and microenvironment response but no cytotoxic T-cell activation in classic Hodgkin lymphoma treated with anti-PD1. Blood. 2020;136(25):2851-2863.

18. Duhrsen U, Muller S, Hertenstein B, et al. Positron Emission Tomography-Guided Therapy of Aggressive Non-Hodgkin Lymphomas (PETAL): a multicenter, randomized phase III trial. J Clin Oncol. 2018;36(20):2024-2034.

19. Tilly H, Gomes da Silva M, Vitolo U, et al. Diffuse large B-cell lymphoma (DLBCL): ESMO clinical practice guidelines for diagnosis, treatment and follow-up. Ann Oncol. 2015;26(Suppl 5):v116-125.

20. Pandey S, Mourcin F, Marchand T, et al. IL-4/CXCL12 loop is a key regulator of lymphoid stroma function in follicular lymphoma. Blood. 2017;129(18):2507-2518.

21. Barraclough A, Bishton M, Cheah CY, Villa D, Hawkes EA. The diagnostic and therapeutic challenges of grade $3 \mathrm{~B}$ follicular lymphoma. Br J Haematol. 2021;195(1):15-24.

22. Klapper W. Pathobiology and diagnosis of follicular lymphoma. Semin Diagn Pathol. 2011;28(2):146-160.

23. Mann RB, Berard CW. Criteria for the cytologic subclassification of follicular lymphomas: a proposed alternative method. Hematol Oncol. 1983;1(2):187-192.

24. Ott G, Katzenberger T, Lohr A, et al. Cytomorphologic, immunohistochemical, and cytogenetic profiles of follicular lymphoma: 2 types of follicular lymphoma grade 3 . Blood. 2002;99(10):3806-3812.

25. Salaverria I, Philipp C, Oschlies I, et al. Translocations activating IRF4 identify a subtype of germinal center-derived B-cell lymphoma affecting predominantly children and young adults. Blood. 2011;118(1):139-147.

26. Klapper W, Kreuz M, Kohler CW, et al. Patient age at diagnosis is associated with the molecular characteristics of diffuse large Bcell lymphoma. Blood. 2012;119(8):1882-1887.

27. Au-Yeung RKH, Arias Padilla L, Zimmermann M, et al. Experience with provisional WHO-entities large B-cell lymphoma with IRF4rearrangement and Burkitt-like lymphoma with 11q aberration in paediatric patients of the NHL-BFM group. Br J Haematol. 2020;190(5):753-763. 